\title{
Solving Fixed Channel Allocation using Hybrid Evolutionary Method
}

\author{
Raja Marappan ${ }^{\mathrm{a}}$ and Gopalakrishnan Sethumadhavan \\ Department of Computer Applications, School of Computing, SASTRA University, Thanjavur, India
}

\begin{abstract}
Assigning channels to cells in wireless networks is an NP-hard problem. There are different soft computing strategies are applied to solve fixed channel allocation with the interference constraints of the mobile network. This research focuses on applying the new genetic operators with the local search and heuristic strategies to obtain the near optimal solution. This hybrid evolutionary method is implemented on some of the benchmark instances. Near optimal solution is obtained in the minimal complexity and the results are found to be better than the existing methods.
\end{abstract}

Keywords- Fixed channel allocation, genetic algorithm, cellular networks, evolutionary method

\section{Introduction}

To increase the performance of wireless networks, the right assignment of frequencies or channels to the cells is to be performed. Based on the interference constraints of the mobile network, allocation should be done to maximize the network bandwidth efficiency. Fixing $\mathrm{m}$ channels to the $\mathrm{n}$ cells is the Fixed Channel Allocation Problem (FCAP), an NP-hard problem [1], [2], [3]. Fixing the channels to cells based on co-channel, adjacent channel and co-site channel constraints is a graph coloring problem. Several new strategies of coloring have been designed to solve large scale graphs to find the near optimal solution [5], [6], [7], [8], [9]. These new strategies are applied in this research to find the solution to FCAP.

The mathematical model of channel allocation is defined in Section 2. The proposed hybrid evolutionary method is presented in Section 3. The simulation results of some of the benchmark instances with the comparison of existing methods are presented in Section 4; and the last Section 5 concludes this research.

\section{Mathematical Model Formulation of FCAP}

For the wireless network with $\mathrm{m}$ channels and $\mathrm{n}$ cells, FCAP is the constraint based minimization problem. The solution $F$ is represented as the binary matrix Fjk such that its elements assume either 0 or 1 .

$$
f_{j k}=\left\{\begin{array}{l}
1 \\
0
\end{array} \text { if channel } k \text { is }\left\{\begin{array}{l}
\text { assigned } \\
\text { not assigned }
\end{array}\right\} \text { to cell } j .\right.
$$

The structure of $\mathrm{F}$ is defined in Fig. 1.

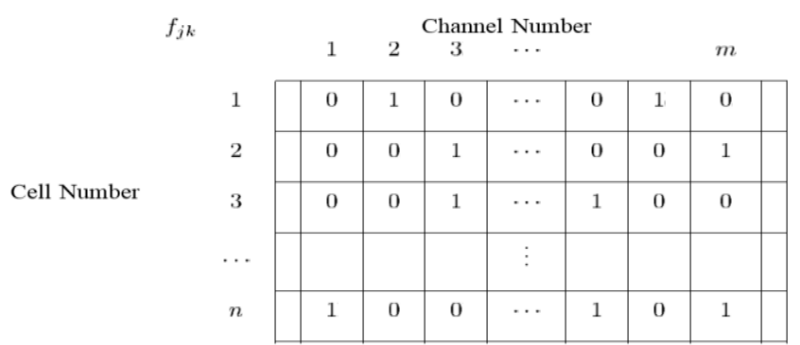

Figure 1. Structure of F.

FCAP defines certain constraints:

Demand d, the first constraint, is defined over F. For cell $\mathrm{j}$, the total number of channels to be fixed is $d_{\mathrm{j}}$. Clearly, the number of assigned 1's in each row should not exceed its cell demand $\mathrm{d}$. When the demand constraint is violated for the $i^{\text {th }}$ cell, then

$$
\left(\sum_{q=1}^{m} f_{i q}-d_{i}\right) \neq 0
$$

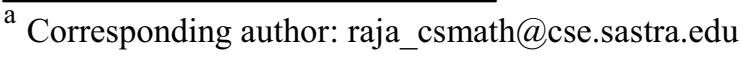


CSC, co-site constraint is the second constraint is defined for channels $\mathrm{p}$ and $\mathrm{q}$ for cell $\mathrm{i}$. For channels $\mathrm{p}$ and $\mathrm{q}$, if $\mid \mathrm{p}$ $\mathrm{q} \mid<\mathrm{c}_{\mathrm{ii}}$, then $\mathrm{i}$ cannot be assigned $\mathrm{p}$. If fixing $\mathrm{p}$ to $\mathrm{i}$ violate this constraint then

$$
\sum_{\substack{q=p-\left(c_{i k}-1\right) \\ q \neq p \\ 1 \leq q \leq m}}^{p+\left(c_{i i}-1\right)} f_{i q}>0
$$

CCC \& ACC, co-channel \& adjacent channel constraints, for channels $\mathrm{p}$ and $\mathrm{q} \&$ cells $\mathrm{i}$ and $\mathrm{j}$, if $|\mathrm{p}-\mathrm{q}|<\mathrm{c}_{\mathrm{ii}}$,

$\mathrm{c}_{\mathrm{ij}}>0, \mathrm{i} \neq \mathrm{j}$, then $\mathrm{i}$ cannot fix $\mathrm{p}$. If these constraints are violated then

$$
\sum_{\substack{j=1 \\ j \neq i \\ c_{i j}>0}}^{n} \sum_{\substack{q=p-\left(c_{i j}-1\right) \\ 1 \leq q \leq m}}^{p+\left(c_{i j}-1\right)} f_{j q}>0
$$

$\mathrm{C}(\mathrm{F})$, the cost function is defined as

$$
\begin{aligned}
C(F)= & \sum_{i=1}^{n} \sum_{p=1}^{m}\left(\sum_{\substack { j=1 \\
j \neq i \\
c_{i j}>0 \\
\begin{subarray}{c}{q=p-\left(c_{i j}-1\right) \\
1 \leq q \leq m{ j = 1 \\
j \neq i \\
c _ { i j } > 0 \\
\begin{subarray} { c } { q = p - ( c _ { i j } - 1 ) \\
1 \leq q \leq m } }\end{subarray}}^{n} f_{j q}\right) f_{i p} \\
& +\sum_{i=1}^{n+\left(c_{i j}-1\right)} \sum_{p=1}^{m}\left(\sum_{\substack{p=p-\left(c_{i i}-1\right) \\
q \neq p \\
1 \leq q \leq m}}^{p+\left(c_{i i}-1\right)} f_{i q}\right) f_{i p} \\
& +\sum_{i=1}^{n}\left(\sum_{q=1}^{m} f_{i q}-d_{i}\right)
\end{aligned}
$$

When these defined constraints are satisfied then $\mathrm{C}(\mathrm{F})$ reaches zero. Hence the FCAP is finding the vector $\mathrm{F}$ so that $\mathrm{C}(\mathrm{F})=0$.

\section{Hybrid Evolutionary Algorithm}

The hybrid evolutionary algorithm with the new genetic operators and local search, heuristic strategies are presented in this section.

The genetic algorithm starts with the initial population, where the initial population is generated using the heuristics and recursive backtracking, $(\lambda, \mu)$ evolution strategies defined in [7], [9]. The initial population is generated to satisfy the demand constraints. Then fitness proportionate selection is applied to select two better and one worst individual as defined in [5]. The selected parents are applied constraint crossover operation with the symmetry-breaking method defined in [8]. The crossover offspring are applied constraint mutation with the symmetry-breaking method defined in [8]. This procedure is repeated for fixed generations to obtain the near optimal solution by performing the elitism operation. The flowchart of this hybrid evolutionary method to solve FCAP is shown in Fig. 2.

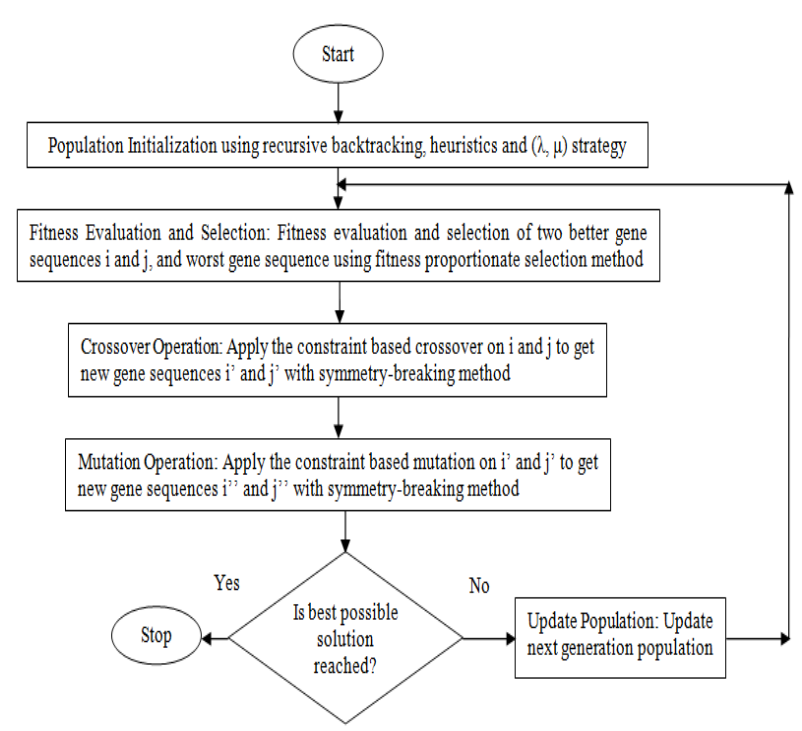

Figure 1. Flowchart of Hybrid Evolutionary Method to solve FCAP.

The constraint based crossover and mutations are defined as follows:

Algorithm Constraint-Crossover (i, j, n, m)

// offspring i' and j' will be returned

Apply the single parent crossover operation defined in [6] for the selected gene sequences $\mathrm{i} \& \mathrm{j}$ to optimize demand and co-site constraints;

Apply the multi parent crossover operation for fixed iterations to fine tune the offspring;

Return i' and j';

Algorithm Constraint-mutation (i', j', n, m)

// offspring $i "$ and $j "$ will be returned

Apply the mutation operation defined in [6] for the offspring i' \& j' to optimize co-channel \& adjacent channel constraints;

Optimize the demand and co-site constraints for fixed number of iterations;

Return i" and j";

Theorem 1: The hybrid evolutionary algorithm achieves stochastic convergence.

Proof: For any two sequences of genes $i, j \in$ finite search space $\mathrm{S}$, then $\mathrm{j}$ is reachable from $\mathrm{i}$.

Since 0

$<$ probability $\left\{\mathrm{j}^{\prime},=\right.$ (Constraint-crossover $(\mathrm{j}) \quad \&$ Constraint-mutation(j')) $\}<1$, clearly reachable condition is fulfilled. Also the population of gene sequences fulfills monotone property [5], [6]. Hence the theorem. 


\section{Simulation of Proposed Hybrid Evolutionary Method and Comparisons}

This hybrid evolutionary method is simulated on some of the benchmark problems using Intel Xeon Workstation with 256 GB DDR3 in Windows 8 Professional OS under JDK 1.8.0 environment. The output performances and comparison with existing approaches [1], [2], [3], [4] are presented here. The Frequency of Convergence (FOC) and fitness threshold for some of the benchmark instances are shown in Table 1. The comparison with existing methods is shown in Table 2. It has been experimentally observed that FOC reaches $100 \%$ for the defined fitness threshold values and the proposed method performs well than the existing methods to obtain the near optimal convergence. For the random graphs of problem [4] \& [5], 100\% FOC threshold is obtained for the lower fitness thresholds. It shows that the hybrid genetic operators achieve stochastic convergence with reduced complexity.

Table 1. Convergence of near optimal solution for benchmark instances.

\begin{tabular}{|c|c|c|c|c|c|}
\hline $\begin{array}{c}\text { Probl } \\
\text { em }\end{array}$ & $\begin{array}{c}\text { No. of } \\
\text { Cells } \\
\text { (n) }\end{array}$ & $\begin{array}{c}\text { No. of } \\
\text { Channe } \\
\text { ls (m) }\end{array}$ & $\begin{array}{c}\text { Simulatio } \\
\mathbf{n} \\
\text { Run } \\
\text { Length }\end{array}$ & $\begin{array}{c}\text { Fitnes } \\
\text { s } \\
\text { Thres } \\
\text { hold }\end{array}$ & $\begin{array}{c}\text { FOC } \\
\text { Thresho } \\
\text { ld }\end{array}$ \\
\hline 1 & 4 & 11 & 100 & 0 & $100 \%$ \\
\hline 2 & 25 & 73 & 25000 & 7 & $100 \%$ \\
\hline 3 & 21 & 385 & 30000 & 0 & $100 \%$ \\
\hline 4 & 500 & 1250 & 50000 & 15 & $100 \%$ \\
\hline 5 & 1000 & 1500 & 75000 & 21 & $100 \%$ \\
\hline
\end{tabular}

Table 2. Comparison with Existing Methods

\begin{tabular}{|c|c|c|c|c|}
\hline \multirow{2}{*}{ Problem } & $\begin{array}{c}\text { Previous } \\
\text { Method }\end{array}$ & \multicolumn{3}{|c|}{ Proposed Method } \\
\cline { 2 - 5 } & FOC & FOC & $\begin{array}{c}\text { No. of } \\
\text { Trials }\end{array}$ & $\begin{array}{c}\text { CPU } \\
\text { Time(sec } \\
\text { ) }\end{array}$ \\
\hline 1 & $100 \%$ & $100 \%$ & 1 & 0 \\
\hline 2 & $92 \%$ & $100 \%$ & 5286 & 781 \\
\hline 3 & $80 \%$ & $100 \%$ & 1 & 0 \\
\hline 4 & - & $100 \%$ & 7501 & 1273 \\
\hline 5 & - & $100 \%$ & 9012 & 2761 \\
\hline
\end{tabular}

\section{Conclusion}

The hybrid constraint based genetic operators with local and recursive search strategies are designed and simulated on some of the FCAP's. The local and recursive backtracking, symmetric breaking and constraint based crossover and mutations significantly reduces the computational complexity to achieve probabilistic convergence. Simulation outcomes show that FOC reaches $100 \%$ for the lower fitness threshold values, and the proposed method performs well than the existing methods to obtain the near optimal convergence. For the random graphs of large sizes also, 100\% FOC threshold is obtained for the lower fitness thresholds. It shows that the constraint genetic operators reach stochastic convergence with minimal complexity.

\section{Acknowledgment}

The authors would like to acknowledge the support rendered by the management of SASTRA University by the way of providing necessary infrastructure and financial support for this research. The authors would like to thank Gary Lewandowski and Michael Trick for uploading the graph repository in World Wide Web Consortium.

\section{Bibliography}

1. Shih Heng Cheng, Ching Yao Huang, ColoringBased Inter-WBAN Scheduling for Mobile Wireless Body Area Networks, IEEE Transactions on parallel and distributed systems, vol. 24, no. 2, 2013.

2. Elmahdi Driouch, Wessam Ajib, Downlink Scheduling and Resource Allocation for Cognitive Radio MIMO Networks, IEEE Transactions on vehicular technology, vol. 62, no. 8, 2013.

3. Elmahdi Driouch, Wessam Ajib, Efficient Scheduling Algorithms for Multiantenna CDMA Systems, IEEE Transactions on vehicular technology, vol. 61, no. 2, 2012.

4. Matti Peltomäki, Juha-Matti Koljonen, Olav Tirkkonen, Mikko Alava, Algorithms for SelfOrganized Resource Allocation in Wireless Networks, IEEE transactions on vehicular technology, vol. 61, no. 1, 2012.

5. Raja Marappan, Gopalakrishnan Sethumadhavan, A new genetic algorithm for graph coloring, In CIMSim2013, 5th International Conference on Computational Intelligence, Modelling and Simulation, Seoul, South Korea, pages 49-54, 2013.

6. Gopalakrishnan Sethumadhavan, Raja Marappan, A Genetic Algorithm for Graph Coloring using Single Parent Conflict Gene Crossover and Mutation with Conflict Gene Removal Procedure, 2013 IEEE International Conference on Computational Intelligence and Computing Research, pp.350-355, 2013.

7. Raja Marappan, Gopalakrishnan Sethumadhavan, Solution to Graph Coloring Problem using Heuristics and Recursive Backtracking, International Journal of Applied Engineering Research, Volume 10, No.10(2015) pp. 2593925944.

8. Raja Marappan, Gopalakrishnan Sethumadhavan, Solution to Graph Coloring Problem using Evolutionary Optimization through Symmetry Breaking Approach, International Journal of Applied Engineering Research, Volume 10, No.10(2015) pp. 26573-26580.

9. Raja Marappan, Gopalakrishnan Sethumadhavan, Solving Graph Coloring Problem for Large Graphs, Global Journal of Pure and Applied Mathematics, volume 11(4), pp. 2487-2494, 2015. 